\title{
Adjunctive corticosteroids may be associated with better outcome for non-HIV Pneumocystis pneumonia with respiratory failure: a systemic review and meta-analysis of observational studies
}

Lin Ding ${ }^{1}$, Huixue Huang ${ }^{2}$, Heyan Wang ${ }^{3}$ and Hangyong He $\mathrm{He}^{4,5,6^{*}}$

\begin{abstract}
Background: Evidence supporting corticosteroids adjunctive treatment (CAT) for Pneumocystis jirovecii pneumonia (PCP) in non-HIV patients is highly controversial. We aimed to systematically review the literature and perform a metaanalysis of available data relating to the effect of CAT on mortality of PCP in non-HIV patients.

Methods: We searched Pubmed, Medline, Embase, and Cochrane database from 1989 through 2019. Data on clinical outcomes from non-HIV PCP were extracted with a standardized instrument. Heterogeneity was assessed with the $R^{2}$ index. Pooled odds ratios and 95\% confidence interval were calculated using a fixed effects model. We analyzed the impact of CAT on mortality of non-HIV PCP in the whole PCP population, those who had hypoxemia $\left(\mathrm{PaO}_{2}<70 \mathrm{mmHg}\right)$ and who had respiratory failure $\left(\mathrm{PaO}_{2}<60 \mathrm{mmHg}\right)$.

Results: In total, 259 articles were identified, and 2518 cases from 16 retrospective observational studies were included. In all non-HIV PCP cases included, there was an association between CAT and increased mortality (odds ratio, $1.37 ; 95 \%$ confidence interval $1.07-1.75 ; P=0.01$ ). CAT showed a probable benefit of decreasing mortality in hypoxemic non-HIV PCP patients (odds ratio, $0.69 ; 95 \%$ confidence interval $0.47-1.01 ; P=0.05$ ). Furthermore, in a subgroup analysis, CAT showed a significantly lower mortality in non-HIV PCP patients with respiratory failure compared to no CAT (odds ratio, $0.63 ; 95 \%$ confidence interval $0.41-0.95 ; P=0.03$ ).

Conclusions: Our meta-analysis suggests that among non-HIV PCP patients with respiratory failure, CAT use may be associated with better clinical outcomes, and it may be associated with increased mortality in unselected non-HIV PCP population. Clinical trials are needed to compare CAT vs no-CAT in non-HIV PCP patients with respiratory failure. Furthermore, CAT use should be withheld in non-HIV PCP patients without hypoxemia.
\end{abstract}

Keywords: Corticosteroids adjunctive treatment (CAT), Pneumocystis pneumonia (PCP), Non-HIV, Respiratory failure

*Correspondence: yonghang2004@sina.com

${ }^{\dagger}$ Lin Ding, Huixue Huang and Heyan Wang are co-first authors

${ }^{6}$ Department of Respiratory and Critical Care Medicine, Beijing

Institute of Respiratory Medicine, Beijing Chao-Yang Hospital, Capital Medical University, No. 8 Gongren Tiyuchang Nanlu, Chaoyang District, Beijing 100020, China

Full list of author information is available at the end of the article

\section{Introduction}

Pneumocystis jirovecii pneumonia (PCP) is a major cause of acute respiratory failure and death in immunocompromised patients. Malignant disease with chemotherapy, corticosteroids treatment for inflammatory autoimmune diseases, and transplantation of solid organs or bone marrow are the leading causes of $\mathrm{T}$ cell suppression, which 
is associated with a high risk of opportunistic infections, including PCP $[1,2]$. The mortality associated with PCP in the non-HIV population group remains high, ranging from 19 to $76 \%$ [3-5], despite the availability of effective antimicrobial agents such as trimethoprim/sulfamethoxazole (TMP-SMZ) and pentamidine.

Early studies done in HIV-positive patients showed that corticosteroids adjunctive treatment (CAT) was associated with a dramatic decrease in mortality during PCP episodes $[6,7]$. The pathophysiology of PCP may differ between patients with and without HIV infection. Conceivably, these differences between the two populations might affect the ability of steroids therapy to provide therapeutic benefits [8].

Alternative therapies such as the use of adjunctive agents in an effort to reduce the mortality associated with non-HIV population have been evaluated by several retrospective studies. Unfortunately, the data for CAT among the non-HIV population are highly controversial $[5,9]$, and one study even indicated potential harm with CAT in severe cases admitted into the intensive care unit (ICU) [10].

Based on relatively limited and controversial data from studies available at this stage, the primary objective of this investigation was to systematically review the literature and perform a meta-analysis of available data relating to evaluation of whether CAT can reduce mortality in non-HIV patients with PCP.

\section{Methods}

\section{Data source and study selection}

A literature search of Pubmed, Medline, Embase, and Cochrane database from 1989 to September 2019 was performed to find published articles evaluating adjunctive corticosteroids therapy for patients with nonHIV PCP. We limited studies to human subjects and searched for the following terms: (Pneumocysti*[text word] OR PCP[text word] OR "Pneumocystis Infections"[MESH] OR "Pneumocystis jirovecii"[MESH] OR "Pneumonia, Pneumocystis"[MESH] OR "Pneumocystis carinii" [MESH]) AND (treat" [text word] OR adjunct" [text word] OR treatment* [text word] OR steroid*[text word] OR corticosteroid*[text word] OR glucocorticoid"[text word] OR "Glucocorticoids"[MESH] OR "Adrenal Cortex Hormones"[MESH] OR "Steroids" [MESH]) NOT (("animals" $[\mathrm{MeSH}] \quad$ NOT "humans" [MeSH]) AND ("non-HIV" OR "non-AIDS" OR "non-HIV-infected" OR "HIV-uninfected" OR "AIDS uninfected" OR "HIV-negative")). In addition, we also reviewed the references listed in the identified articles and performed a manual search of the related articles to identify all relevant and eligible articles and to minimize publication bias.
Two researchers screened and evaluated the eligibility of all studies independently, and a third reviewer intervened whenever there was a disagreement. The inclusion criteria were (1) original research report of PCP in non-HIV patients; (2) report of mortality from patients with non-HIV PCP; (3) reported with data on mortality between patients treated with and without adjunctive corticosteroids, and (4) written in English. The exclusion criteria were (1) written in languages other than English; (2) not related to PCP pneumonia; (3) not an original research; (4) in vitro studies; (5) reports of single case experiences. The intervention of interest was corticosteroids adjunctive therapy. The comparison groups were patients treated for non-HIV PCP with and without adjunctive corticosteroids. The outcome of interest was mortality in all non-HIV PCP patients and mortality in those with acute hypoxemic respiratory failure, as defined by the study investigators. The meta-analysis of observational studies in epidemiology criteria were used to conduct this investigation, and the article follows MOOSE criteria [11].

\section{Data extraction}

Two investigators extracted data independently from each eligible study, using a standardized data extraction form. Discrepancies in data extraction underwent arbitration by a third reviewer, and consensus was obtained by verbal discussion. Data collected from each study included author, year of study, country, age group of patients, number of patients, causes of immunosuppression, method of PCP diagnosis, treatment of PCP, definition of adjunctive corticosteroid use, dose of steroids used, and mortality.

\section{Data synthesis and data analysis}

Data on non-HIV PCP outcomes were collected from all articles. All-cause mortality and clinical cure rates, as defined by the individual studies, were the primary outcome measures used in this meta-analysis. Odds ratios for mortality were calculated for each article. The significance level for this meta-analysis model was set at $P<0.05$. Forest plots were provided for mortality in this meta-analysis. We used the $\mathrm{Q}$ statistic to test the existence of heterogeneity; a $P$ value of less than 0.10 was considered significant for heterogeneity. $I^{2}$ was employed to assess the proportion of total variability due to heterogeneity. An $I^{2}$ value of approximately $25 \%$ was regarded as low heterogeneity, $50 \%$ as medium, and $75 \%$ as high heterogeneity. Publication bias was assessed and an applicability concerns graph/summary was conducted using Review Manager version 5.3 (Cochrane Collaboration, Oxford, UK). 
For the mortality in all non-HIV PCP patients, we performed a subgroup meta-analysis on different types of mortality reported (ICU mortality, hospital mortality, 30-day mortality and 60-day mortality). For the mortality in patients with hypoxemia or respiratory failure, we performed a subgroup meta-analysis on different severity of hypoxemia: hypoxemia patients with a $\mathrm{PaO}_{2}<70 \mathrm{mmHg}$ (including patients with $60 \mathrm{mmHg}<\mathrm{PaO}_{2}<70 \mathrm{mmHg}$ and patients with $\mathrm{PaO}_{2}<60 \mathrm{mmHg}$ ), and respiratory failure patients with a $\mathrm{PaO}_{2}<60 \mathrm{mmHg}$. For the different doses of CAT used, we performed a subgroup meta-analysis on different patients with high dose ( $>240 \mathrm{mg}$ prednisone equivalent) or low dose $(1 \mathrm{mg} / \mathrm{kg} /$ day prednisone equivalent) CAT.

\section{Results}

\section{Literature search}

The literature search yield a total of 259 articles possibly related to non-HIV PCP with CAT. By reviewing the titles and abstracts, studies were mainly excluded due to the following: not an original research $(n=46)$; reports of single case experiences $(n=35)$; only diagnostic studies $(n=33)$; only HIV patients $(n=24)$; written in languages other than English $(n=22)$; not related to PCP pneumonia $(n=15)$; not related to corticosteroids therapy $(n=13)$; in vivo/in vitro studies $(n=5)$. After the initial screening, 66 articles with data on treatment of non-HIV PCP remained for full-text review. Of these, 50 articles were excluded from further analysis because they did not include outcome data $(n=43)$, reports of PCP colonization without evidence of infection $(n=5)$, or duplicated reports $(n=2)$. In the final analysis, 16 retrospective cohort investigations of patients treated with CAT were included [3-5, 9, 12-23] (Fig. 1).

\section{Study characteristics}

Demographic variables of the included studies are summarized in Table 1. A total of 16 studies involving 2518 patients were included in this meta-analysis. The 16 studies were conducted in 7 countries as France $(n=4)$, Japan $(n=4)$, United States $(n=2)$, China $(n=2)$, Korea $(n=2)$, Italy $(n=1)$, and Greece $(n=1)$.

The type of immunocompromised diseases of non-HIV patient population included hematological malignancies $(n=715)$, solid tumor $(n=421)$, rheumatoid arthritis $(n=257)$, solid organ transplantation $(n=133)$, and other inflammatory autoimmune diseases $(n=393$, including collagen vascular disease, interstitial lung disease, connective tissue disease) (Table 1).

Diagnosis of PCP was based on methenamine silver stain under microscopic examination (7 studies), fluorescent antibody staining (8 studies), or polymerase chain reaction (4 studies) of the patients' sputum/bronchoalveolar lavage fluid, or transbronchial biopsy (1 study), or elevated concentration of plasma 1,3-D-glucan (1 study) (Table 1).

The included investigations differed in their definition of CAT from different centers (shown in Table 1). The investigations defined CAT use as prednisone doses greater than $60 \mathrm{mg} /$ day or methylprednisolone $50-80 \mathrm{mg} /$ day, pulse corticosteroids(high dose corticosteroids as $240 \mathrm{mg} /$ day for 3 days, with the dosage then tapered during the next 10 days, until no therapy was given), prednisone doses of $80 \mathrm{mg}$ or greater, and prednisone doses $>1 \mathrm{mg} / \mathrm{kg}$.

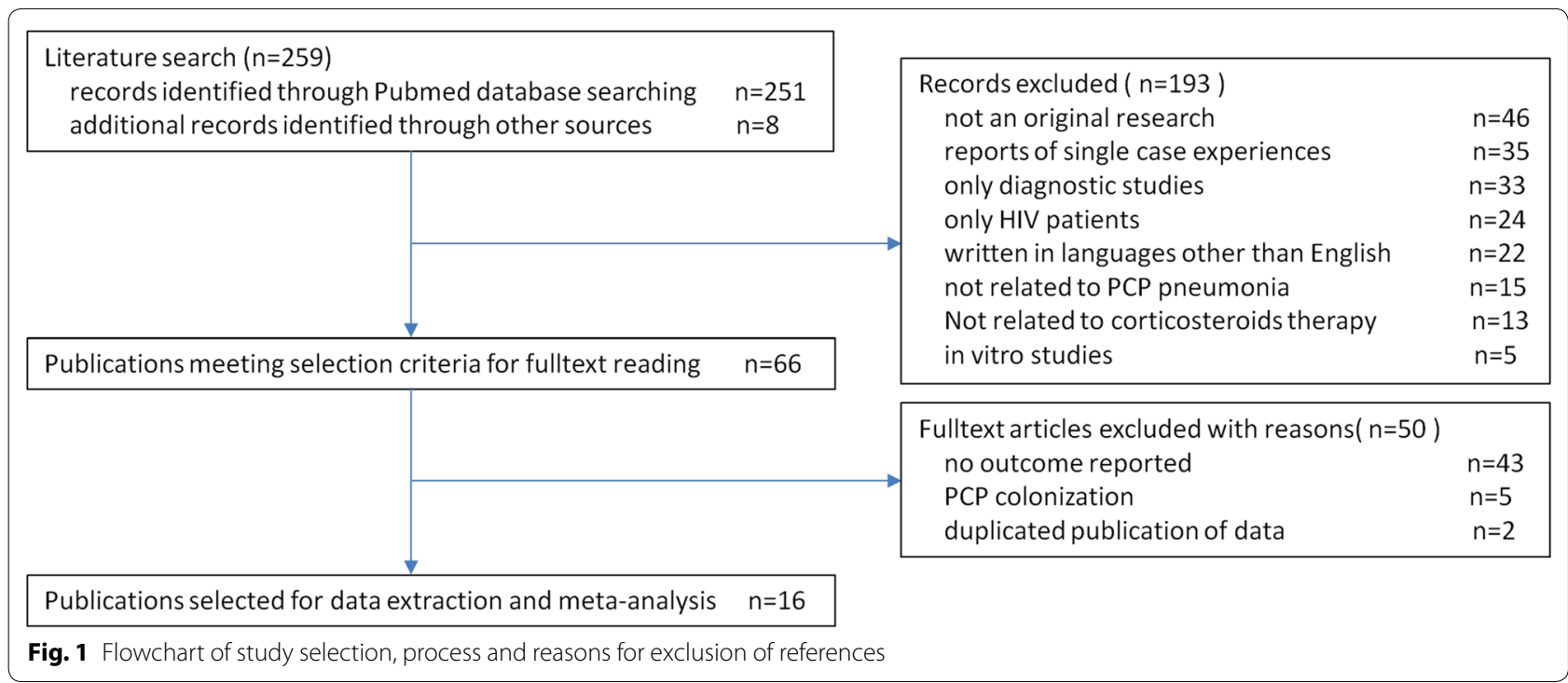




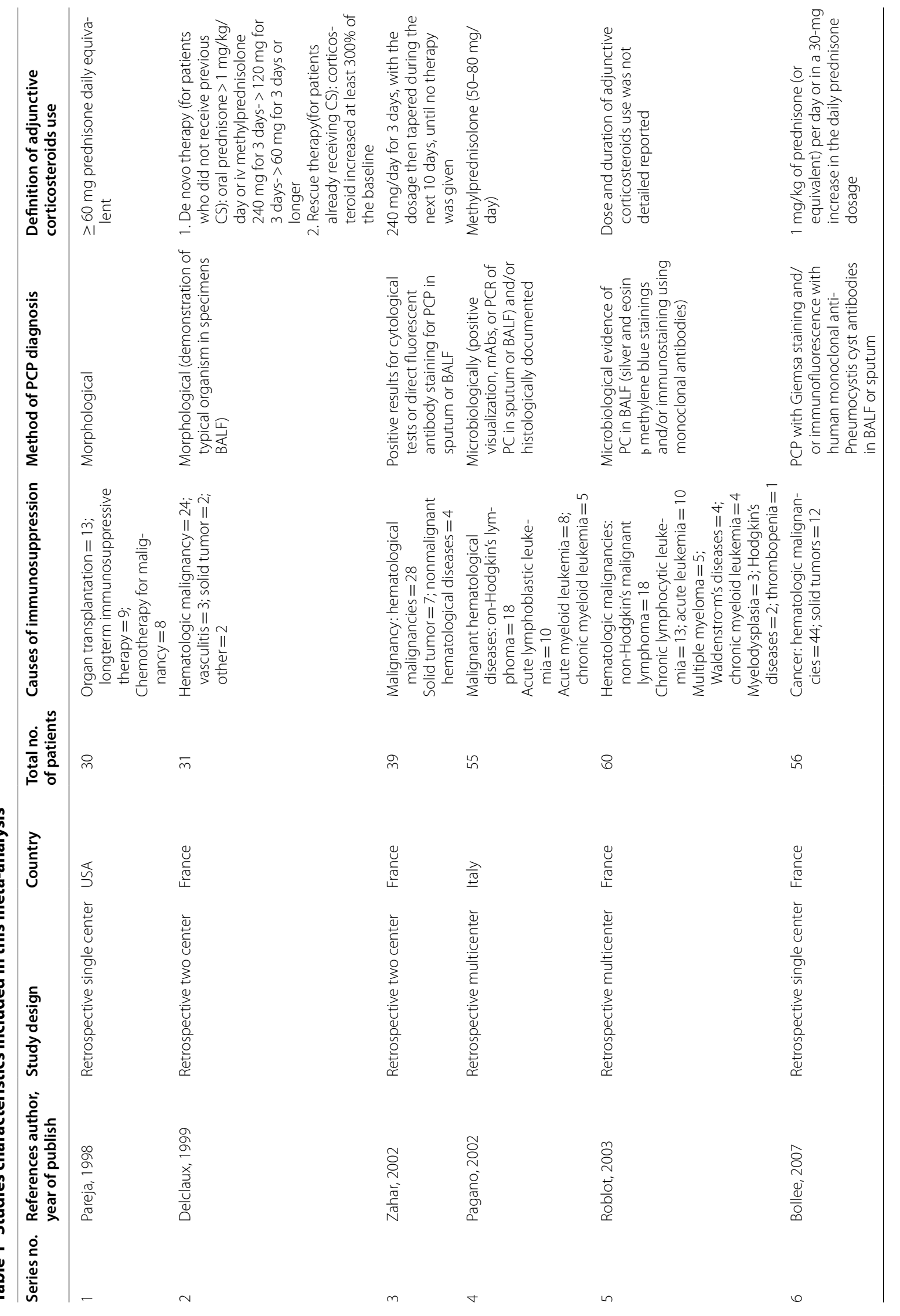




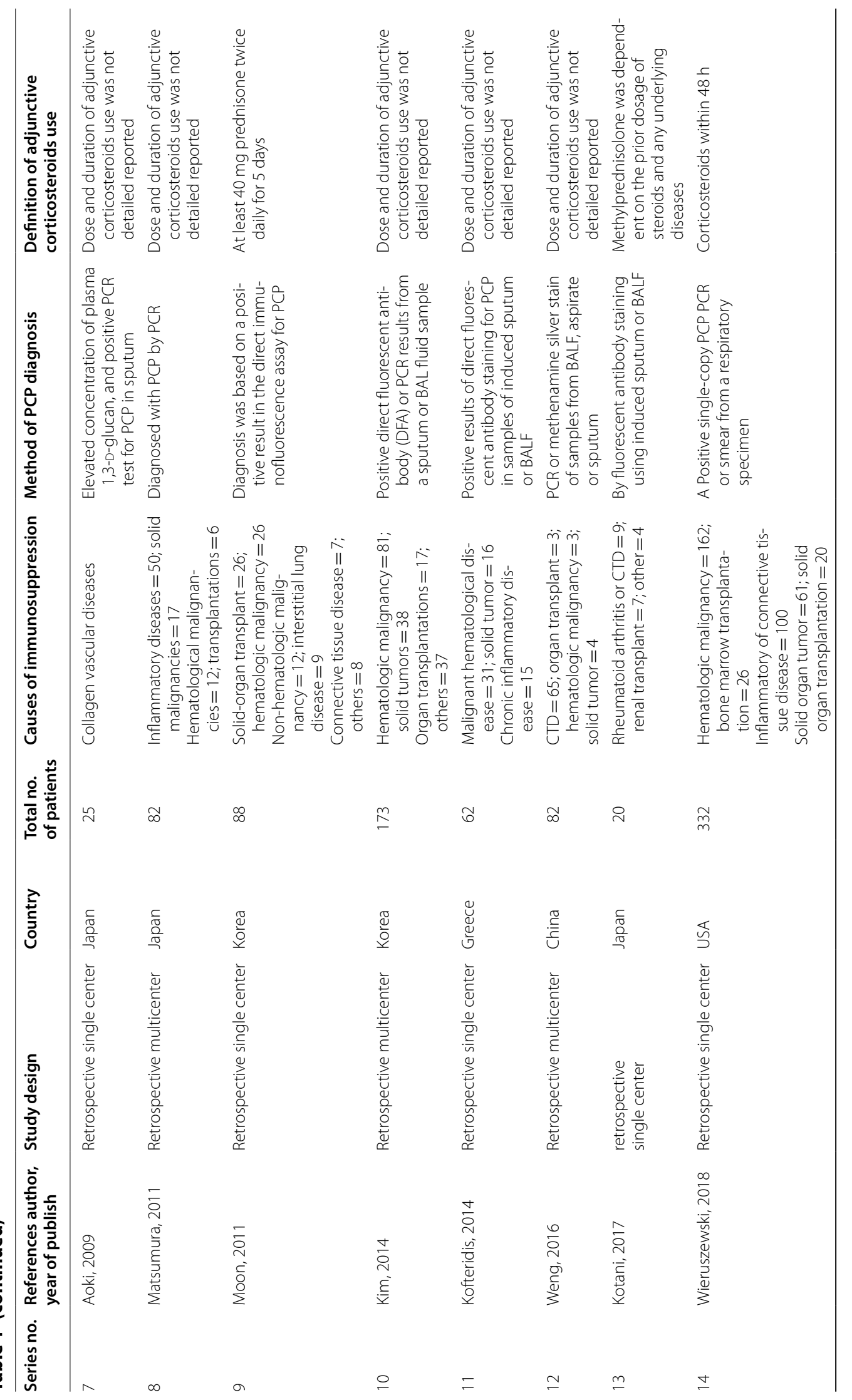


Studies also differed in their definition of mortality (Table 2). Six investigations used in-hospital mortality rates, six investigations used 30 day all-cause mortality, two used 60 day all-cause mortality, and two used ICU mortality rates.

\section{Impact of corticosteroids adjunctive therapy on mortality of non-HIV PCP}

We performed a meta-analysis on mortality associated with or without CAT in 16 studies in all non-HIV PCP patients (Table 2). Figure 2 shows treatment with CAT for non-HIV PCP patients have an significant impact on mortality (odds ratio, 1.37; 95\% confidence interval $1.07-1.75 ; P=0.01)$. The CAT was related with a higher mortality in all non-HIV PCP patients included.

Subgroup analysis for different types of mortality were performed (as shown in Additional file 1: Figure 2.2). Treatment with CAT for non-HIV PCP patients had no significant impact on ICU mortality (odds ratio, 0.77; 95\% confidence interval 0.29-2.08; $P=0.61$, Additional file 1: Figure 2.2.1) and 60-day mortality (odds ratio, 0.97; 95\% confidence interval $0.67-1.41 ; P=0.89$, Additional file 1: Figure 2.2.4). Treatment with CAT for non-HIV PCP patients had a significant impact on hospital mortality (odds ratio, 2.14; 95\% confidence interval 1.17-2.91; $P=0.01$, Additional file 1: Figure 2.2.2) and 30-day mortality (odds ratio, 1.85; 95\% confidence interval 1.20-2.84; $P=0.005$, Additional file 1: Figure 2.2.3).

Impact of corticosteroids adjunctive therapy on mortality of non-HIV PCP with hypoxemia or respiratory failure

We performed another meta-analysis on mortality associated with or without CAT in 8 studies in which nonHIV PCP patients with hypoxemia or acute hypoxemic respiratory failure were reported (Table 2). Figure 3 shows CAT was related to a trend of lower mortality for non-HIV PCP patients with hypoxemia (odds ratio, 0.69; $95 \%$ confidence interval $0.47-1.01 ; P=0.05$ ).

Subgroup analysis for hypoxemia or respiratory failure was performed (as shown in Fig. 4). Treatment with CAT for non-HIV PCP patients with hypoxemia had no significant impact on mortality (odds ratio, 1.06; 95\% confidence interval 0.44-2.57; $P=0.89$, Fig. 4a). Treatment with CAT for non-HIV PCP patients with respiratory failure had a significant impact on mortality (odds ratio, 0.63 ; $95 \%$ confidence interval $0.41-0.95 ; P=0.03$, Fig. 4b). CAT was related to a significantly lower mortality for non-HIV PCP patients with respiratory failure. 


\section{Impact of high- or low-dose corticosteroids adjunctive} therapy on mortality of non-HIV PCP

High dose (>240 mg prednisone equivalent) or low dose $(1 \mathrm{mg} / \mathrm{kg} /$ day prednisone equivalent) CAT were reported in 7 studies in non-HIV PCP patients. We performed another meta-analysis on mortality associated with or without CAT in different CAT doses. Figure 5 shows CAT dose had no relation with a lower mortality for non-HIV PCP patients (odds ratio, 0.92; 95\% confidence interval $0.68-1.25 ; P=0.59$ ).

Subgroup analysis for hypoxemia patients with high or low dose CAT were performed (as shown in Fig. 6). Treatment with low dose CAT for non-HIV PCP patients with hypoxemia had a significant impact on mortality (odds ratio, 0.61; 95\% confidence interval, $0.39-0.94 ; P=0.02$, Fig. 6a). Treatment with high dose CAT for non-HIV PCP patients with hypoxemia had no significant impact on mortality (odds ratio, 0.79; $95 \%$ confidence interval 0.16-4.00; $P=0.78$, Fig. 6b). Low dose CAT was related to a significantly lower mortality for non-HIV PCP patients with hypoxemia.

\section{Subgroup analysis of studies published before or after 2005}

Based on over 20 years of publication range (from 1998 to 2019) of included studies, all studies published after 2005 had an inclusion period after 2000, which may represent different ventilation strategies (lung protective ventilation for ARDS) applied to severe PCP pneumonia induced respiratory failure. Subgroup analysis for the impact of publication range on studies was performed.

In subgroup analysis of 11 studies published after 2005, treatment with CAT for non-HIV PCP patients had a significant impact on mortality (odds ratio, 1.39; 95\% confidence interval 1.07-1.75; $P=0.01$, Fig. 7b). In subgroup analysis of five studies with hypoxemia or respiratory failure published after 2005, Fig. 8b shows CAT was related to a significantly lower mortality for non-HIV PCP patients with hypoxemia (odds ratio, 0.64 ; $95 \%$ confidence interval $0.42-0.96 ; P=0.03$ ).

\section{Publication bias}

More than eight studies reported mortality in all studies and in studies with hypoxemia patients, so we used funnel plot analysis to assess publication bias. As shown in Additional file 2, there was no obvious evidence of publication bias for mortality in all studies and in studies with hypoxemia patients by funnel plots.

\section{Discussion}

Our systematic review and meta-analysis revealed for the first time that CAT is harmful in unselected nonHIV PCP patients with or without respiratory failure, but may reduce mortality in the subgroup of non-HIV PCP patients who have acute hypoxemic respiratory failure. The main strength of our study is that it had the largest sample size of 2518 cases from 16 studies of non-HIV PCP patients treated with or without CAT.

Firstly, our analysis found that when evaluated in the whole non-HIV PCP population, CAT showed an association with higher mortality. This result indicated that CAT may increase the mortality in unselected PCP patients, including those with or without hypoxemia. This finding is different from that in the HIV-infected population. In a large RCT for HIV-PCP [7], clinical benefit of early CAT could not be demonstrated for HIVinfected patients with mild hypoxemia (a partial pressure of oxygen greater than $75 \mathrm{mmHg}$ on room air), but CAT also showed no harm for mortality. In the most recent two large sample studies in non-HIV PCP, there was no difference in mortality between CAT and no CAT $[4,5]$.

Secondly, our analysis found a probable association between CAT and low mortality in non-HIV PCP patients with hypoxemia or respiratory failure $(\mathrm{OR}=0.69,95 \% \mathrm{CI} 0.47-1.01, P=0.05)$. This result is similar to findings from the HIV-infected patients. Prospective studies in hypoxemic HIV patients with PCP found that early initiation of corticosteroids reduced the need for mechanical ventilation and conferred the greatest mortality benefit $[6,7,24,25]$. Moon et al. [19] evaluated CAT in moderate to severe non-HIV PCP, and also indicated that CAT may not improve the outcome of PCP in non-HIV patients. However, when considering the heterogeneity of the present analysis $\left(I^{2}=47 \%\right)$, the different treatment protocols between studies and the long time elapsed between the different studies can lead to wide differences in the prognosis of critically ill patients, including mechanically ventilated patients. Furthermore, as for the $P=0.05$, this result could not be considered for a conclusive negative recommendation of CAT use in non-HIV PCP with respiratory insufficiency. The negative result may be due to the design of this analysis, which only included retrospective observational studies, and used different definitions in time and dose of CAT use, which led to heterogenous effects to the outcome.

Thirdly, our analysis found an association between CAT and low mortality in non-HIV PCP patients with respiratory failure $\left(\mathrm{PaO}_{2}<60 \mathrm{mmHg}\right)$. Limited studies reported the effects of CAT on mortality of non-HIV PCP with respiratory failure. Pareja et al. [9] described that the adjunctive corticosteroids shortened the duration of the mechanical ventilation and may accelerate 


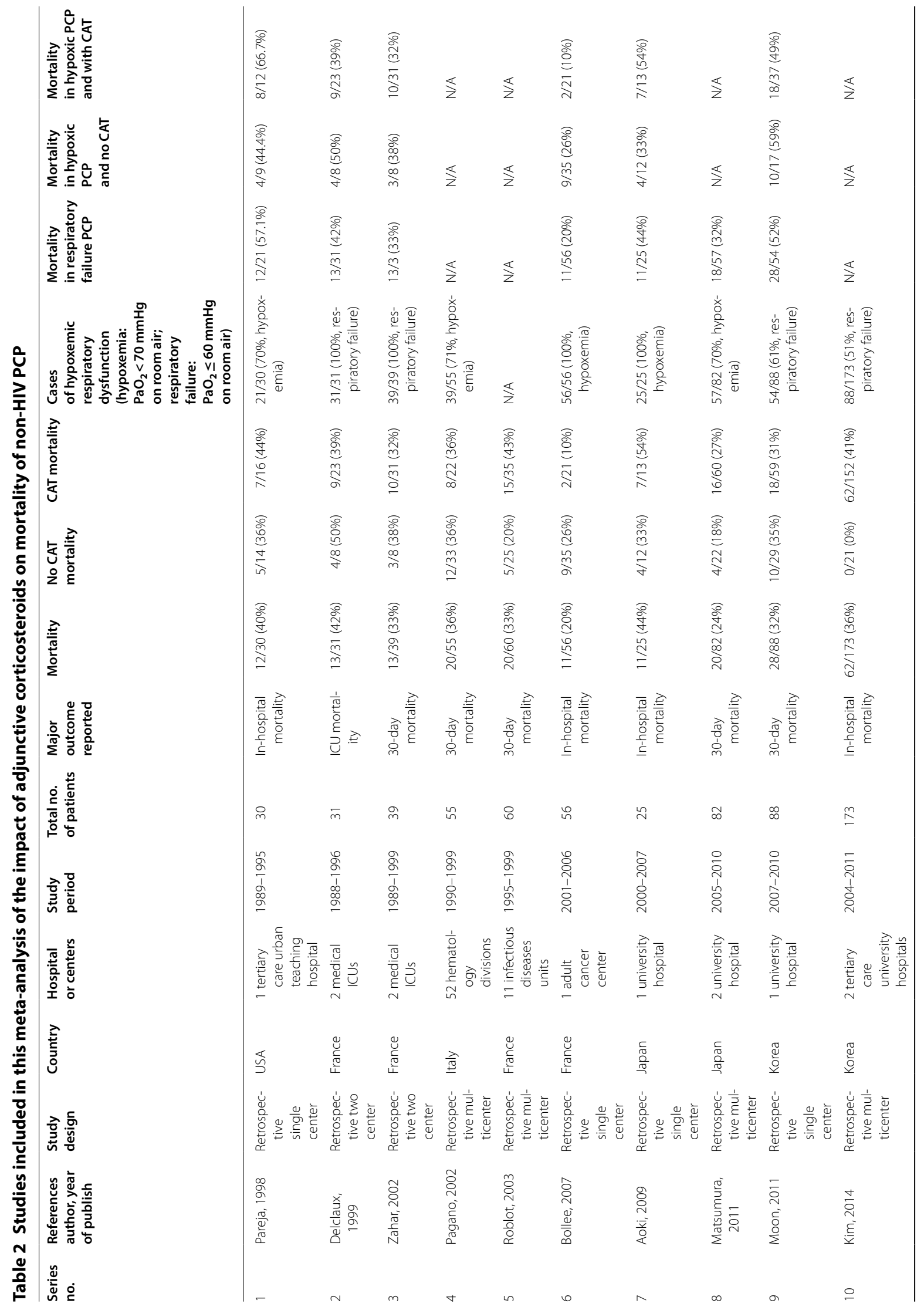




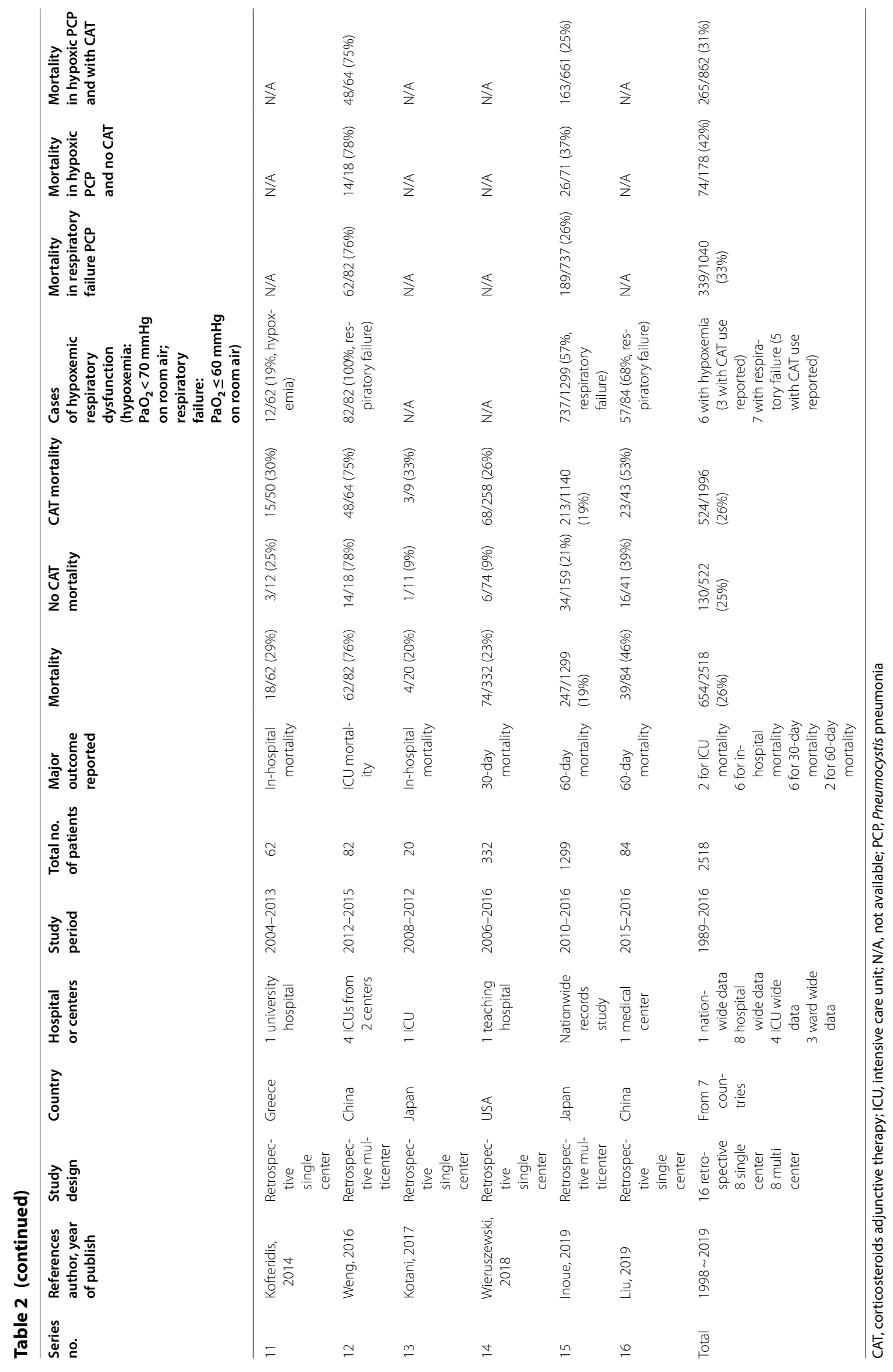




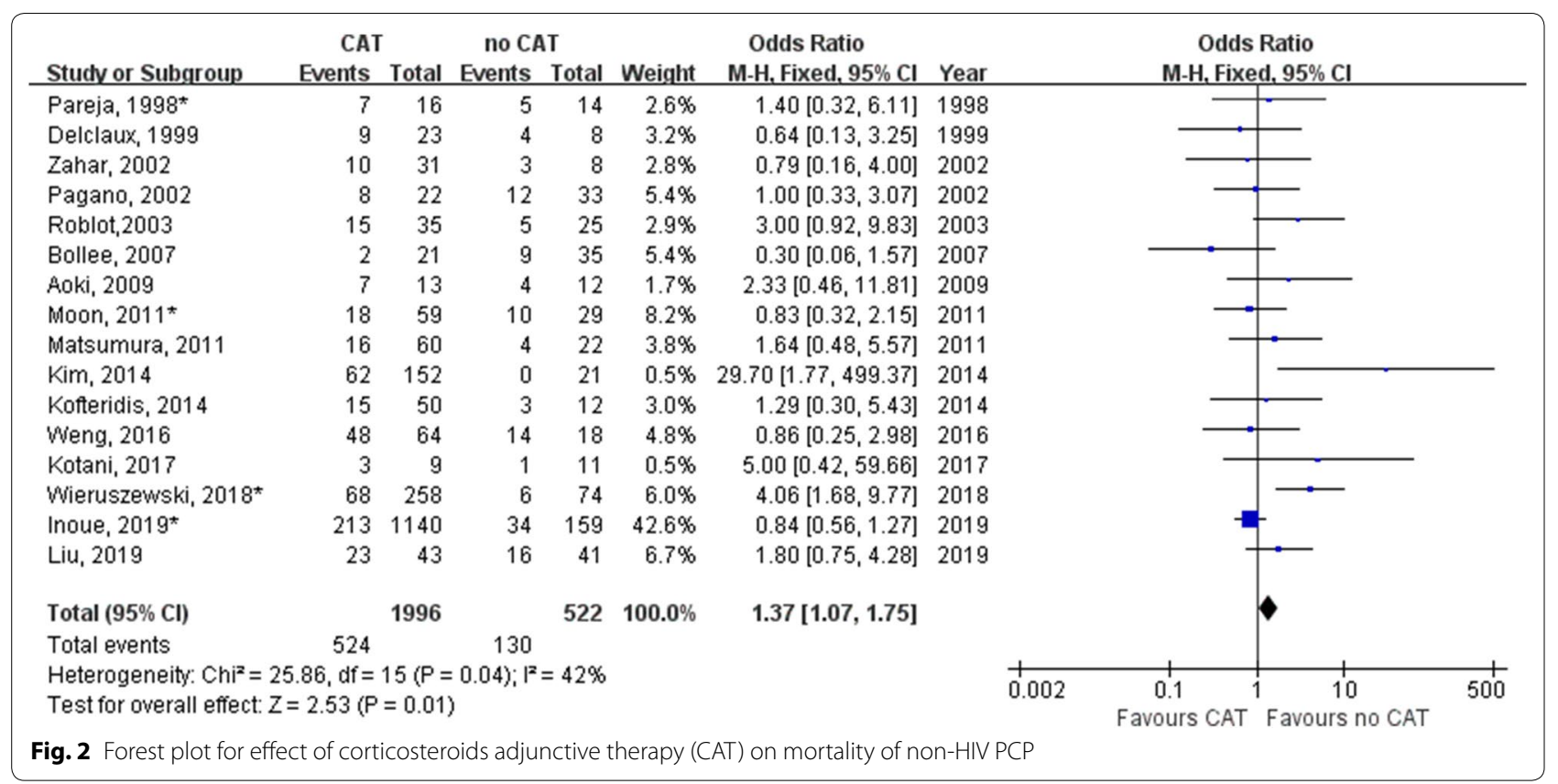

\begin{tabular}{|c|c|c|c|c|c|c|c|c|c|c|}
\hline \multirow{2}{*}{$\begin{array}{l}\text { Studv or Subgroup } \\
\text { Pareia } 1998^{*}\end{array}$} & \multirow{2}{*}{$\begin{array}{r}\text { CAT } \\
\text { Events } \\
8\end{array}$} & \multirow{2}{*}{$\frac{\text { Total }}{12}$} & $\begin{array}{l}\text { no-CA } \\
\text { Events }\end{array}$ & Total & Weight & $\begin{array}{c}\text { Odds Ratio } \\
\text { M-H. Fixed, } 95 \% \mathrm{Cl}\end{array}$ & \multirow{2}{*}{$\frac{\text { Year }}{1998}$} & \multicolumn{3}{|c|}{$\begin{array}{c}\text { Odds Ratio } \\
\text { M-H, Fixed, } 95 \% \mathrm{Cl}\end{array}$} \\
\hline & & & 4 & 9 & $2.4 \%$ & $2.50[0.42,14.83]$ & & & & \\
\hline Delclaux, 1999 & 9 & 23 & 4 & 8 & $5.6 \%$ & $0.64[0.13,3.25]$ & 1999 & & & \\
\hline Zahar, 2002 & 10 & 31 & 3 & 8 & $5.0 \%$ & $0.79[0.16,4.00]$ & 2002 & & & \\
\hline Bollee, 2007 & 2 & 21 & 9 & 35 & $9.5 \%$ & $0.30[0.06,1.57]$ & 2007 & & & \\
\hline Aoki, 2009 & 7 & 13 & 4 & 12 & $3.0 \%$ & $2.33[0.46,11.81]$ & 2009 & & & \\
\hline Moon, 2011* & 18 & 37 & 10 & 17 & $10.9 \%$ & $0.66[0.21,2.12]$ & 2011 & & & \\
\hline Weng, 2016 & 48 & 64 & 14 & 18 & $8.5 \%$ & $0.86[0.25,2.98]$ & 2016 & & & \\
\hline Inoue, 2019* & 163 & 661 & 26 & 71 & $55.0 \%$ & $0.57[0.34,0.95]$ & 2019 & & & \\
\hline Total (95\% Cl) & & 862 & & 178 & $100.0 \%$ & $0.69[0.47,1.01]$ & & & & \\
\hline Total events & 265 & & 74 & & & & & & & \\
\hline $\begin{array}{l}\text { Heterogeneity: } \mathrm{Chi}^{2}= \\
\text { Test for overall effect: }\end{array}$ & $\begin{array}{l}5.86, d f= \\
Z=1.93\end{array}$ & $\begin{array}{l}7(P= \\
P=0.0\end{array}$ & $\begin{array}{l}0.56) ; 1^{2}= \\
05)\end{array}$ & $0 \%$ & & & & 0.1 & $\begin{array}{cc}1 & 1 \\
0.2 & 0.5 \\
\text { Favours [CAT] }\end{array}$ & $\begin{array}{cccc}1 & 1 & 1 \\
\text { Favours [no-CAT] }\end{array}$ \\
\hline
\end{tabular}

recovery in cases of non-HIV PCP. Although their study suggested potential benefit of using CAT, it showed no difference in mortality. Lemiale et al. [10] focused on the effect of CAT in non-HIV severe PCP admitted to ICU, and found that high dose steroid was even associated with increased mortality. And recently, Inoue et al. [4] analyzed the severe non-HIV PCP group with and without CAT in a nationwide case series, and revealed that CAT was associated with lower risk of 60-day mortality in non-HIV PCP patients with severe respiratory failure. Moreover, even though a low $\mathrm{PaO}_{2}(<60 \mathrm{mmHg})$ is part of the definition of acute respiratory insufficiency, the use of the level of $\mathrm{PaO}_{2}$ alone has some limits to adequately report the severity of Pneumocystis pneumonia. The use of $\mathrm{PaO}_{2} / \mathrm{FiO}_{2}$ may be more informative to this purpose. This may have a great impact on the results of the present analysis. The future analysis should consider $\mathrm{PaO}_{2} / \mathrm{FiO}_{2}$ rather than $\mathrm{PaO}_{2}$ alone to classify the patients. However, because there were fewer number of studies included, and the standard of the hypoxemia or respiratory failure was subjective based on the retrospective observational studies, the results from the subgroup analysis on hypoxemia and respiratory failure was inconclusive at this stage. Although not definitive, this subgroup analysis at least suggested that CAT may have the potential benefit for mortality in patients with hypoxemia and respiratory failure. 


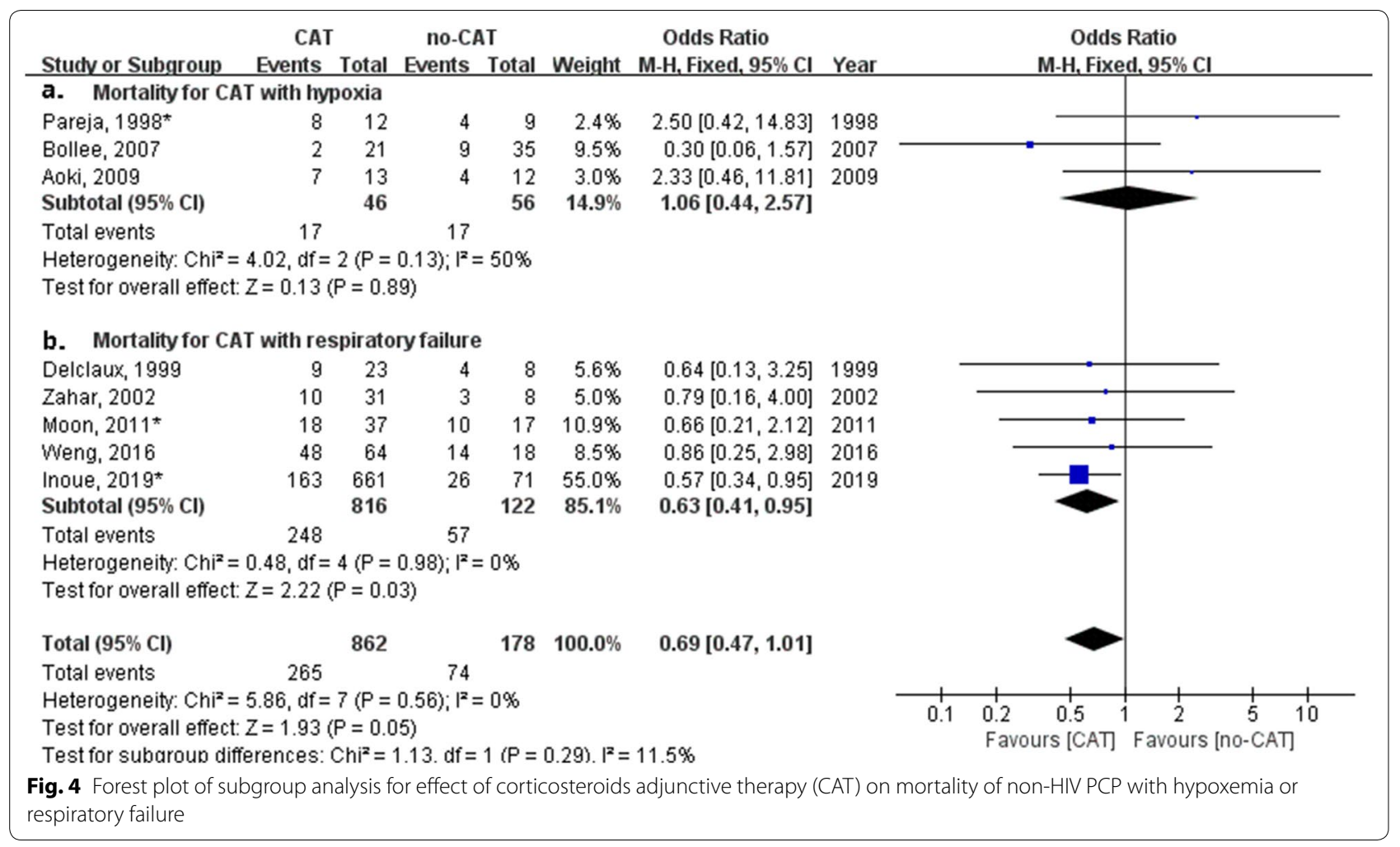

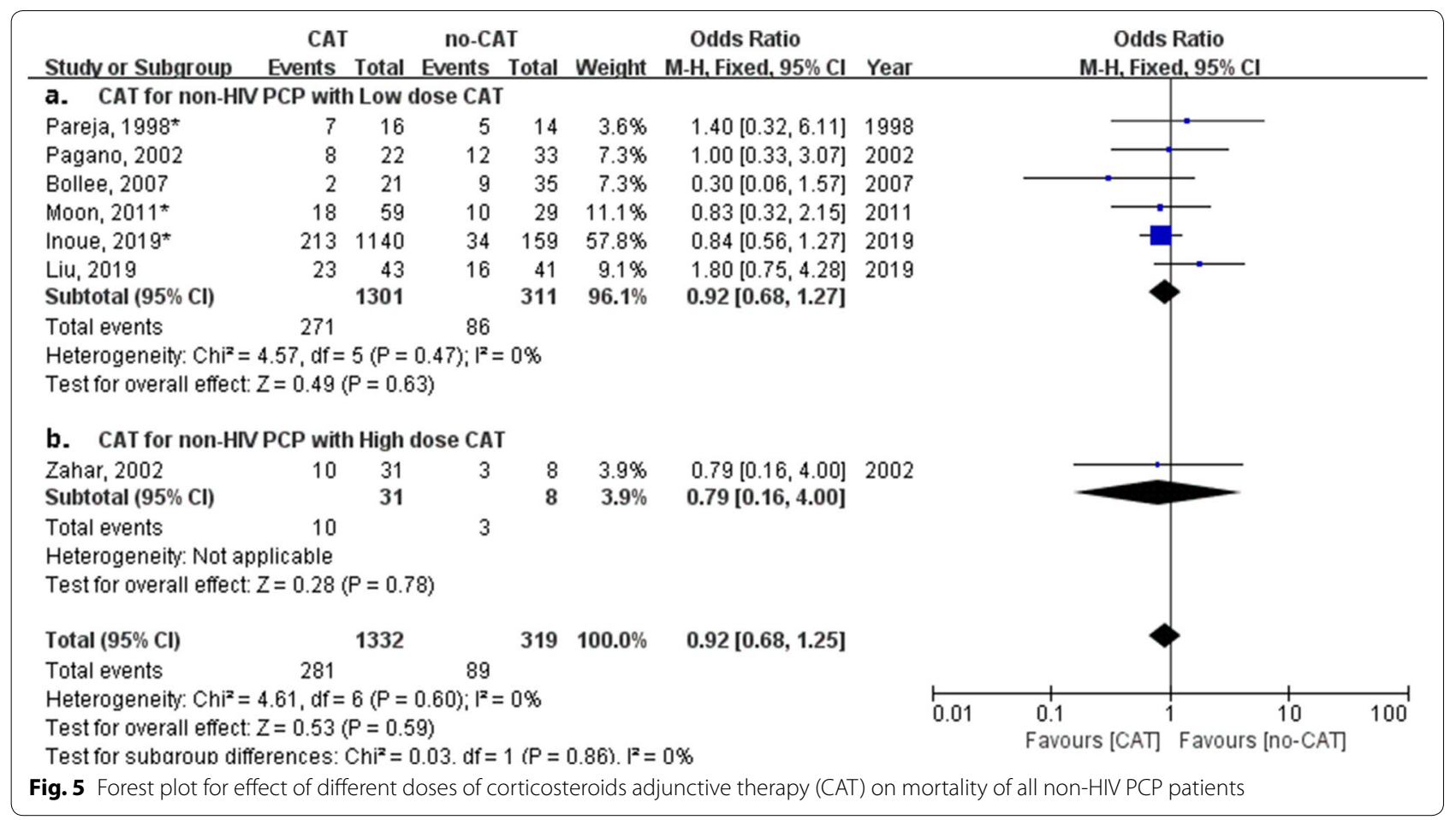

Finally, when we combined the results that CAT increased mortality in unselected non-HIV PCP 


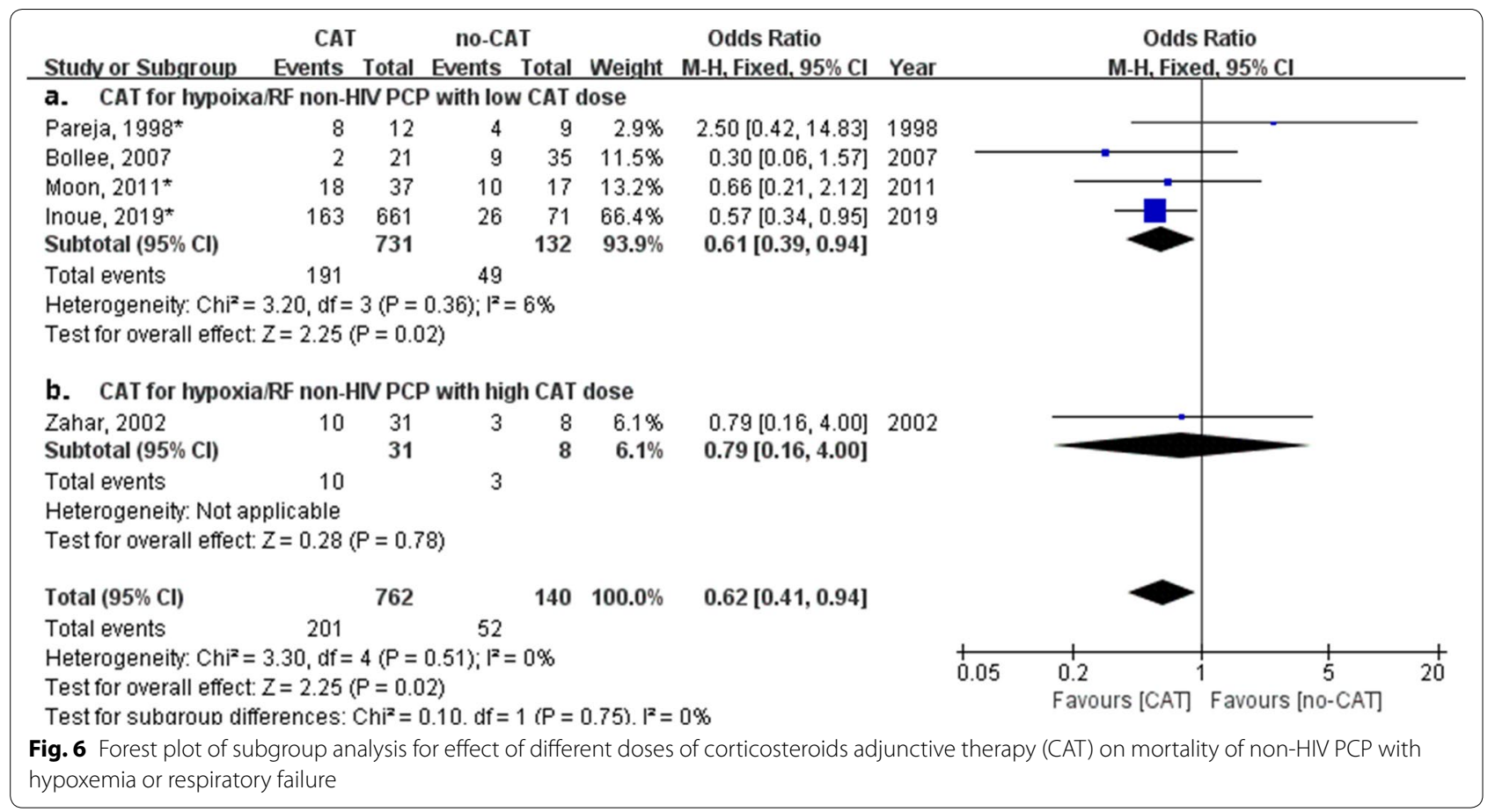

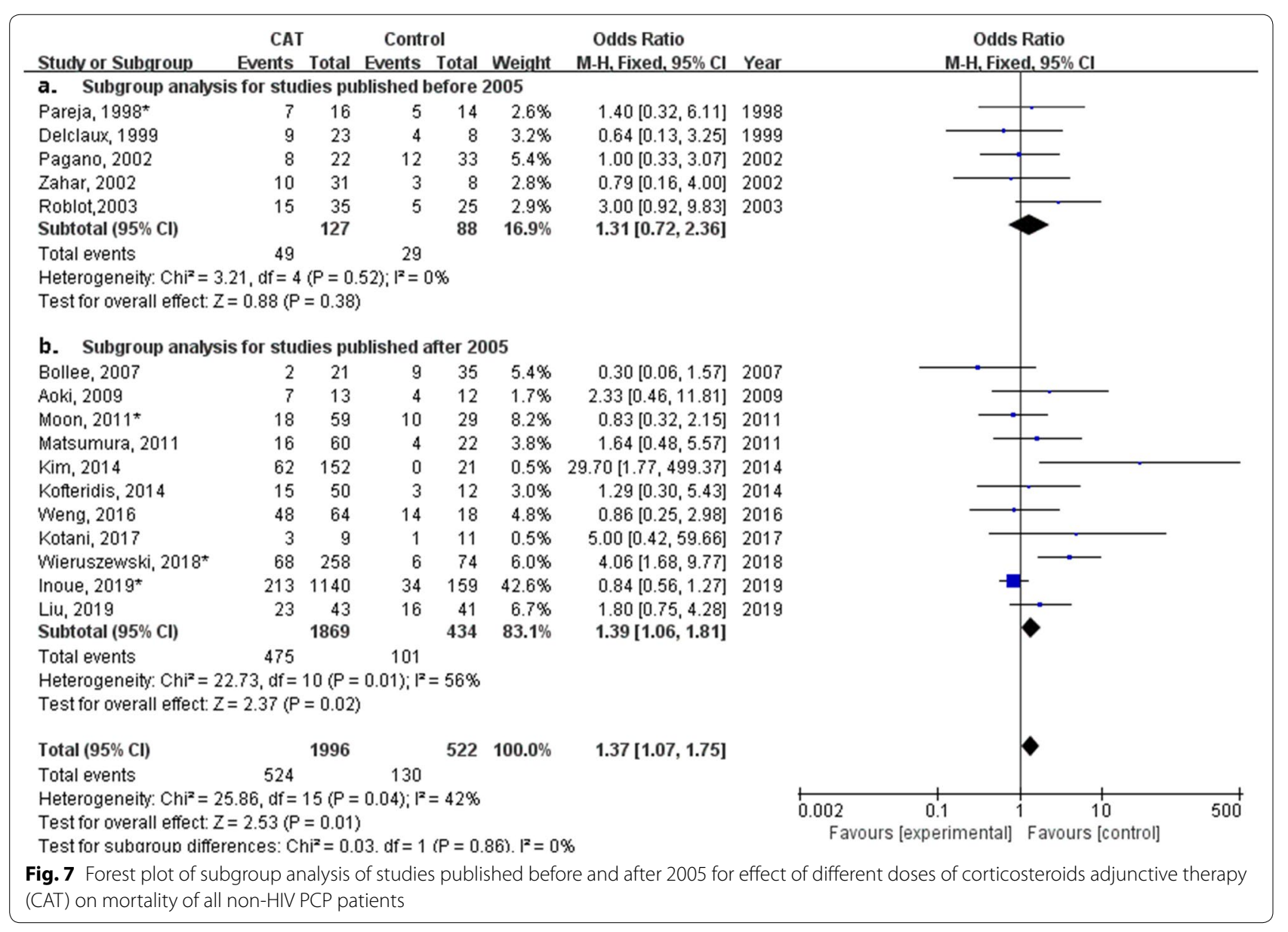




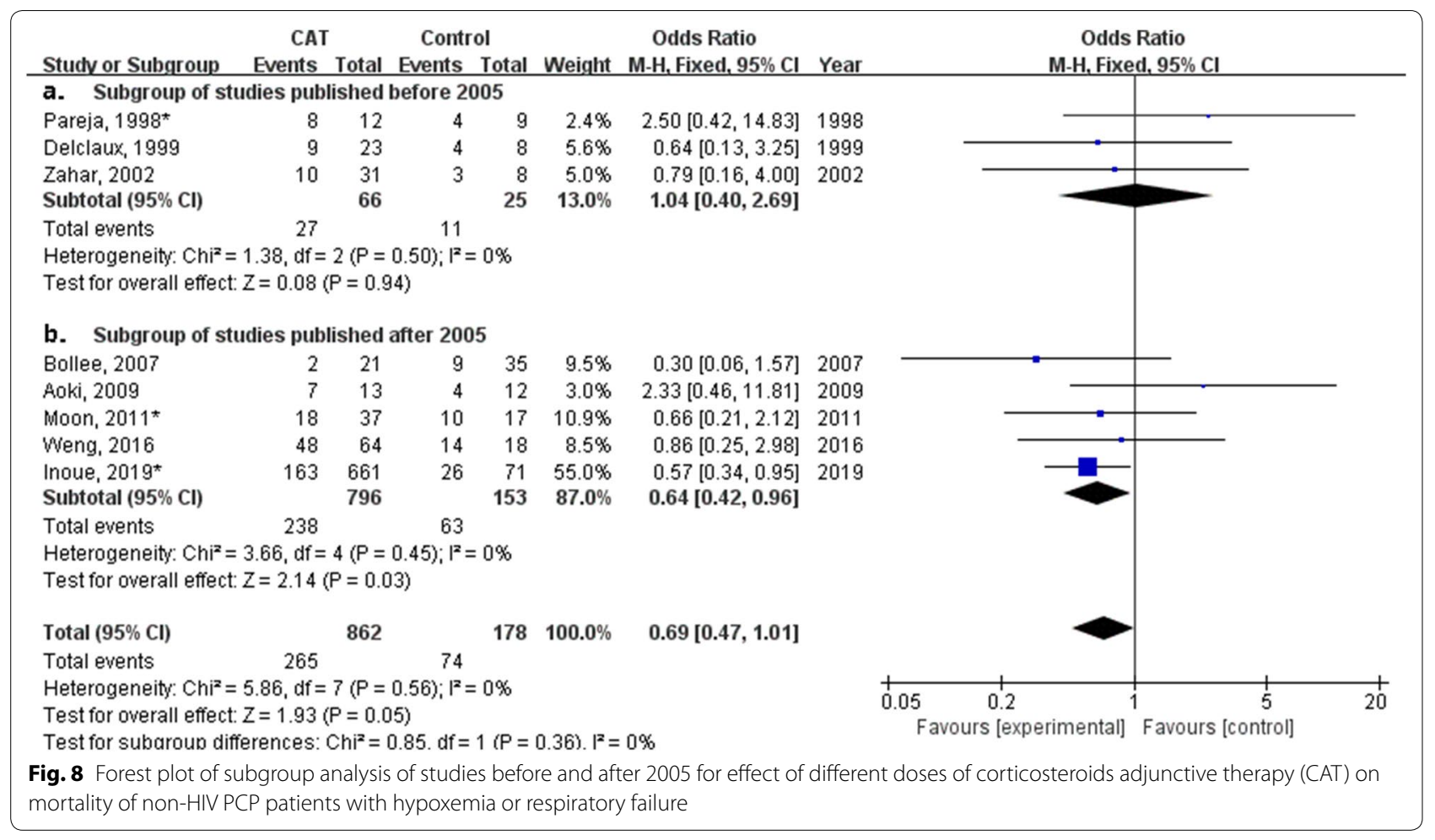

patients, there was a trend of decreasing mortality in moderate and severe PCP patients with hypoxemia, and significantly decreased mortality in patients with respiratory failure, indicating that CAT may be associated with an increased mortality mainly in PCP without hypoxemia, and with a decreased mortality mainly in severe non-HIV PCP with respiratory failure. These findings is different from that in the HIV-PCP $[4,5,7]$. Therefore, based on our analysis, we speculated that CAT may be avoided in non-HIV PCP without hypoxemia, and may be considered in those with respiratory failure.

Moreover, comparing the impact of high and low doses of corticosteroids is of critical importance. Indeed, corticosteroids are in some patients the factor leading to the development of PCP, and low doses may only represent (both in the physician point of view and by its proper effect) the continuation of the treatment instead of an adjunctive therapy. The classification proposed by Lemiale et al. [10] was very meaningful in terms of the dosage of corticosteroids: no corticosteroids, low dose or high dose. In the study by Inoue and coworkers, patients with respiratory failure had received low dose corticosteroids (mean dose of equivalent prednisone in the present study was $39 \mathrm{mg} /$ day), and they found benefits on mortality with low dose (17-36 mg/day) by subgroup of corticosteroids daily dose, a result quite similar to that proposed in Lemiale's study.

Our analysis has limitations. First, all studies included were retrospective studies, no prospective randomized trials were included. The studies included used different definitions in CAT and type of mortality, which led to heterogenous effects on the outcome. Moreover, this meta-analysis was performed on retrospective studies, and timing and dose of steroid in severe patients were not clearly described. However, we did subgroup analysis to explore potential heterogeneity. Second, due to lack of reported data, alternative endpoints other than mortality, such as intubation rate, duration of ventilation, and length of hospital/ICU stay, were unable to be analyzed.

\section{Conclusions}

This systematic review and meta-analysis included 16 studies with 2518 participants, providing an overview of research on CAT for non-HIV PCP. Our meta-analysis suggests that among non-HIV PCP patients with respiratory failure, CAT use may be associated with better clinical outcomes, and it may be associated with increased mortality in unselected non-HIV PCP population. We believe that clinical trials are needed to compare CAT vs no-CAT in non-HIV PCP patients with respiratory failure. Furthermore, CAT use may be withheld in non-HIV PCP patients without hypoxemia. 


\section{Supplementary information}

Supplementary information accompanies this paper at https://doi. org/10.1186/s13613-020-00649-9.

Additional file 1: Figure 2.2. Subgroup analysis for diferent types of mortality.

Additional file 2. Result of publication bias.

\section{Abbreviations}

ICU: Intensive care unit; RF: Respiratory failure; CAT: Corticosteroids adjunctive therapy; PCP: Pneumocystis jirovecii pneumonia.

\section{Acknowledgements}

No.

\section{Authors' contributions}

$\mathrm{LD}, \mathrm{HH}$, and $\mathrm{HW}$ carried out the literature search and data extraction, analyzed the data and wrote the manuscript. HH conceived of the study, and participated in its design and coordination and helped to draft the manuscript. All authors read and approved the final manuscript.

\section{Funding}

None.

\section{Availability of data and materials}

The data can be available in the online database.

\section{Ethics approval and consent to participate}

This study has no ethics approval needed.

\section{Consent for publication}

No need for consent of research and publication.

\section{Competing interests}

The authors declare that they have no competing interests.

\section{Author details}

${ }^{1}$ Department of Respiratory and Critical Care Medicine, Beijing Luhe Hospital, Beijing, China. ${ }^{2}$ Department of Medicine, Beijing University of Technology Hospital, Beijing, China. ${ }^{3}$ Department of Critical Care Medicine, The Sixth Hospital of Guiyang, Guiyang, Guizhou, China. ${ }^{4}$ Beijing Key Laboratory of Respiratory and Pulmonary Circulation Disorders, Beijing, China. ${ }^{5}$ Beijing Engineering Research Center for Diagnosis and Treatment of Pulmonary and Critical Care, Beijing, China. ${ }^{6}$ Department of Respiratory and Critical Care Medicine, Beijing Institute of Respiratory Medicine, Beijing Chao-Yang Hospital, Capital Medical University, No. 8 Gongren Tiyuchang Nanlu, Chaoyang District, Beijing 100020, China.

Received: 19 December 2019 Accepted: 6 March 2020 Published online: 20 March 2020

\section{References}

1. Sepkowitz KA. Pneumocystis carinii pneumonia in patients without AIDS. Clin Infect Dis. 1993;17(Suppl 2):S416-22.

2. Coyle PV, McCaughey C, Nager A, McKenna J, O'Neill H, Feeney SA, Fairley D, Watt A, Cox C, Curran T. Rising incidence of Pneumocystis jirovecii pneumonia suggests iatrogenic exposure of immune-compromised patients may be becoming a significant problem. J Med Microbiol. 2012;61(Pt 7):1009-15.

3. Weng $L$, Huang $X$, Chen L, Feng LQ, Jiang W, Hu XY, Peng JM, Wang CY, Zhan QY, Du B. Prognostic factors for severe Pneumocystis jiroveci pneumonia of non-HIV patients in intensive care unit: a bicentric retrospective study. BMC Infect Dis. 2016;16(1):528.

4. Inoue N, Fushimi K. Adjunctive corticosteroids decreased the risk of mortality of non-HIV Pneumocystis pneumonia. Int J Infect Dis. 2019;79:109-15.
5. Wieruszewski PM, Barreto JN, Frazee E, Daniels CE, Tosh PK, Dierkhising RA, Mara KC, Limper AH. Early corticosteroids for Pneumocystis pneumonia in adults without HIV are not associated with better outcome. Chest. 2018;154(3):636-44.

6. Gagnon S, Boota AM, Fischl MA, Baier H, Kirksey OW, La Voie L. Corticosteroids as adjunctive therapy for severe Pneumocystis carinii pneumonia in the acquired immunodeficiency syndrome. A double-blind, placebocontrolled trial. N Engl J Med. 1990;323(21):1444-50.

7. Bozzette SA, Sattler FR, Chiu J, Wu AW, Gluckstein D, Kemper C, Bartok A, Niosi J, Abramson I, Coffman J, et al. A controlled trial of early adjunctive treatment with corticosteroids for Pneumocystis carinii pneumonia in the acquired immunodeficiency syndrome. California Collaborative Treatment Group. N Engl J Med. 1990;323(21):1451-7.

8. Nuesch R, Bellini C, Zimmerli W. Pneumocystis carinii pneumonia in human immunodeficiency virus (HIV)-positive and HIV-negative immunocompromised patients. Clin Infect Dis. 1999;29(6):1519-23.

9. Pareja JG, Garland R, Koziel H. Use of adjunctive corticosteroids in severe adult non-HIV Pneumocystis carinii pneumonia. Chest. 1998;113(5):1215-24.

10. Lemiale V, Debrumetz A, Delannoy A, Alberti C, Azoulay E. Adjunctive steroid in HIV-negative patients with severe Pneumocystis pneumonia. Respir Res. 2013;14:87.

11. Stroup DF, Berlin JA, Morton SC, Olkin I, Williamson GD, Rennie D, Moher D, Becker BJ, Sipe TA, Thacker SB. Meta-analysis of observational studies in epidemiology: a proposal for reporting. Meta-analysis Of Observational Studies in Epidemiology (MOOSE) group. JAMA. 2000;283(15):2008-12.

12. Delclaux C, Zahar JR, Amraoui G, Leleu G, Lebargy F, Brochard L, Schlemmer B, Brun-Buisson C. Corticosteroids as adjunctive therapy for severe Pneumocystis carinii pneumonia in non-human immunodeficiency virus-infected patients: retrospective study of 31 patients. Clin Infect Dis. 1999:29(3):670-2

13. Zahar JR, Robin M, Azoulay E, Fieux F, Nitenberg G, Schlemmer B. Pneumocystis carinii pneumonia in critically ill patients with malignancy: a descriptive study. Clin Infect Dis. 2002;35(8):929-34.

14. Pagano L, Fianchi L, Mele L, Girmenia C, Offidani M, Ricci P, Mitra ME, Picardi M, Caramatti C, Piccaluga P, et al. Pneumocystis carinii pneumonia in patients with malignant haematological diseases: 10 years' experience of infection in GIMEMA centres. Br J Haematol. 2002;117(2):379-86.

15. Roblot F, Le Moal G, Godet C, Hutin P, Texereau M, Boyer E, PrazuckT, Lacroix C, Souala MF, Raffi F, et al. Pneumocystis carinii pneumonia in patients with hematologic malignancies: a descriptive study. J Infect. 2003:47(1):19-27.

16. Bollee G, Sarfati C, Thiery G, Bergeron A, de Miranda S, Menotti J, de Castro N, Tazi A, Schlemmer B, Azoulay E. Clinical picture of Pneumocystis jiroveci pneumonia in cancer patients. Chest. 2007;132(4):1305-10.

17. Aoki Y, Iwamoto M, Kamata Y, Nagashima T, Yoshio T, Okazaki H, Minota S. Prognostic indicators related to death in patients with Pneumocystis pneumonia associated with collagen vascular diseases. Rheumatol Int. 2009;29(11):1327-30

18. Matsumura $Y$, Shindo $Y$, linuma $Y$, Yamamoto $M$, Shirano $M$, Matsushima A, Nagao M, Ito Y, Takakura S, Hasegawa Y, et al. Clinical characteristics of Pneumocystis pneumonia in non-HIV patients and prognostic factors including microbiological genotypes. BMC Infect Dis. 2011;11:76.

19. Moon SM, Kim T, Sung H, Kim MN, Kim SH, Choi SH, Jeong JY, Woo $\mathrm{JH}$, Kim YS, Lee SO. Outcomes of moderate-to-severe Pneumocystis pneumonia treated with adjunctive steroid in non-HIV-infected patients. Antimicrob Agents Chemother. 2011;55(10):4613-8.

20. Kim SJ, Lee J, Cho YJ, Park YS, Lee CH, Yoon HI, Lee SM, Yim JJ, Lee JH, Yoo CG, et al. Prognostic factors of Pneumocystis jirovecii pneumonia in patients without HIV infection. J Infect. 2014;69(1):88-95.

21. Kofteridis DP, Valachis A, Velegraki M, Antoniou M, Christofaki M, Vrentzos GE, Andrianaki AM, Samonis G. Predisposing factors, clinical characteristics and outcome of Pneumocystis jirovecii pneumonia in HIV-negative patients. J Infect Chemother. 2014;20(7):412-6.

22. Kotani T, Katayama S, Miyazaki Y, Fukuda S, Sato Y, Ohsugi K. Risk factors for the mortality of Pneumocystis jirovecii pneumonia in non-HIV patients who required mechanical ventilation: a retrospective case series study. Biomed Res Int. 2017;2017:7452604

23. Liu CJ, Lee TF, Ruan SY, Yu CJ, Chien JY, Hsueh PR. Clinical characteristics, treatment outcomes, and prognostic factors of Pneumocystis pneumonia in non-HIV-infected patients. Infect Drug Resist. 2019;12:1457-67. 
24. National Institutes of Health-University of California Expert Panel for Corticosteroids as Adjunctive Therapy for Pneumocystis P. Consensus statement on the use of corticosteroids as adjunctive therapy for Pneumocystis pneumonia in the acquired immunodeficiency syndrome. N Engl J Med. 1990;323(21):1500-4.

25. Ewald H, Raatz H, Boscacci R, Furrer $H$, Bucher HC, Briel M. Adjunctive corticosteroids for Pneumocystis jiroveci pneumonia in patients with HIV infection. Cochrane Database Syst Rev. 2015;4:CD006150.

\section{Publisher's Note}

Springer Nature remains neutral with regard to jurisdictional claims in published maps and institutional affiliations.

\section{Submit your manuscript to a SpringerOpen ${ }^{\circ}$ journal and benefit from:}

- Convenient online submission

- Rigorous peer review

- Open access: articles freely available online

- High visibility within the field

- Retaining the copyright to your article

Submit your next manuscript at $\boldsymbol{\nabla}$ springeropen.com 